\title{
The Inhibition of Melanogenesis Via the PKA and ERK Signaling Pathways by Chlamydomonas reinhardtii Extract in B16F10 Melanoma Cells and Artificial Human Skin Equivalents
}

\author{
Ayeong Lee ${ }^{1,2}$, Ji Yea Kim ${ }^{1,2}$, Jina Heo ${ }^{3,4}$, Dae-Hyun Cho ${ }^{3}$, Hee-Sik Kim ${ }^{3,4}$, In-Sook $\mathrm{An}^{2}$, Sungkwan An ${ }^{1}$, and \\ Seunghee $\mathrm{Bae}^{1 *}$ \\ ${ }^{1}$ Research Institute for Molecular-Targeted Drugs, Department of Cosmetics Engineering, Konkuk University, Seoul 05029, Republic of Korea \\ ${ }^{2}$ Korea Institute for Skin and Clinical Sciences, Gene Cell Pharm Corporation, Seoul 05029, Republic of Korea \\ ${ }^{3}$ Sustainable Bioresource Research Center, KRIBB, Daejeon 34141, Republic of Korea \\ ${ }^{4}$ Green Chemistry and Environmental Biotechnology, University of Science and Technology (UST), Daejeon 34113, Republic of Korea
}

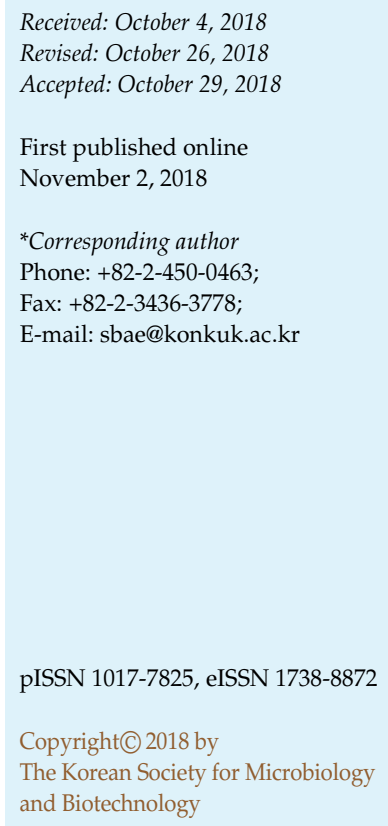

\begin{abstract}
Abnormal melanin synthesis results in several hyperpigmentary disorders such as freckles, melanoderma, age spots, and other related conditions. In this study, we investigated the antimelanogenic effects of an extract from the microalgae Chlamydomonas reinhardtii (CE) and potential mechanisms responsible for its inhibitory effect in B16F10, normal human epidermal melanocyte cells, and human skin-equivalent models. The CE extract showed significant dosedependent inhibitory effects on $\alpha$-melanocyte-stimulating, hormone-induced melanin synthesis in cells. Additionally, the CE extract exhibited suppressive effects on the mRNA and protein expression of microphthalmia-associated transcription factor, tyrosinase, tyrosinaserelated protein-1, and tyrosinase-related protein-2. The CE extract also inhibited the phosphorylation of protein kinase A and extracellular signal-related kinase, which function as upstream regulators of melanogenesis. Using a three-dimensional, reconstructed pigmented epidermis model, the CE-mediated, anti-pigmentation effects were confirmed by FontanaMasson staining and melanin content assays. Taken together, CE extract can be used as an anti-pigmentation agent.
\end{abstract}

Keywords: Chlamydomonas reinhardtii, anti-pigmentation, cell culture, human skin equivalents, melanin

\section{Introduction}

Skin pigmentation is a major physiological defense mechanism against various harmful effects such as ultraviolet (UV)-induced nuclear DNA damage of skin cells. This pigmentation is due to the specialized pigment melanin [1]. Melanin is synthesized and stored within an organelle known as the melanosome and is subsequently transferred to adjacent keratinocytes for distribution throughout the epidermis [2]. Although skin pigmentation plays an essential role in physiological UV protection, abnormal pigmentation such as hyperpigmentation can lead to several dermatological disorders, such as melasma, freckles, age spots, actinic keratosis, and skin cancer [3, 4]. Hyperpigmentation is caused by various biological and environmental factors, including inflammation and UV irradiation; however, effective treatments for pigmentation disorders are based on an anti-pigmentation strategy [4]. Identifying natural compounds that can regulate or inhibit melanogenesis would therefore be valuable for pharmaceutical and biomedical purposes.

Melanogenesis is the metabolic process of melanin production in melanocytes [5]. This process involves a series of enzymatic reactions that convert tyrosine to melanin $[3,5]$. Tyrosinase is the essential and rate-limiting enzyme that catalyzes the conversion of L-tyrosine to 
L-dihydroxyphenylalanine (L-DOPA), which is then converted to L-DOPAquinone, a precursor of melanin [6]. Tyrosinase levels are strongly correlated with pigmentation levels in human skin, indicating that inhibition of tyrosinase activity is a potential anti-pigmentation strategy [7]. The expression level of tyrosinase is modulated by the transcription factor microphthalmia-associated transcription factor (Mitf), a critical factor that regulates skin pigmentation and melanocyte differentiation, proliferation, and survival [8]. In the skin, UV irradiation-induced hyperpigmentation is mediated by $\alpha$-melanocyte-stimulating hormone ( $\alpha-\mathrm{MSH})$ induced Mitf expression [9]. In this pathway, $\alpha-\mathrm{MSH}$ directly binds to the melanocortin-1 receptor (MC1R) on melanocytes [9]. This activates MC1R and causes accumulation of intracellular $\mathrm{CAMP}$, which upregulates the transcription of Mitf by activating the protein kinase A (PKA)/cAMP response element-binding protein (CREB) pathway $[9,10]$. Mitf then binds to the E-box in the tyrosinase promoter region to enhance tyrosinase gene expression [10].

Microalgae are either unicellular or simple multicellular organisms; they grow rapidly and produce several types of secondary metabolites to survive and adapt to highly stressful environments with pressures from extreme temperatures, photo-oxidation, and ultraviolet radiation [11]. During this process, they also produce bioactive compounds that may have desirable effects in cosmetic or pharmaceutical products, such as anti-aging, skin whitening, and anti-pigmentation properties [11, 12]. Chlamydomonas reinhardtii (CE), a species of microalgae, contains functional molecules such as antioxidant agents and pigments including carotenoids and phenolic compounds $[13,14]$. However, the inhibitory effect of CE extract on melanogenesis has not been reported and its molecular mechanism of action remains unclear. In this study, treatment of B16F10 mouse melanoma and normal human epidermal melanocyte (NHEM) cells with CE extract strongly inhibited melanogenesis and significantly suppressed the expression of melanogenesis-related genes. Furthermore, in a threedimensional reconstructed human pigmented epidermis (3D-RHPE) model, repeated topical or systemic applications of $\mathrm{CE}$ extract significantly decreased melanin synthesis. This is the first study to demonstrate the anti-pigmentation effects of CE extract and its potential use in suppressing skin pigmentation.

\section{Materials and Methods}

\section{Preparation of CE Extracts}

CE samples were obtained from the Korea Research Institute of
Bioscience and Biotechnology (KRIBB, Korea). The algae were cultivated in SOT medium (Zarrouk's media) under normal light $\left(200 \mu \mathrm{mol} / \mathrm{m}^{2} / \mathrm{s}\right)$ at $25^{\circ} \mathrm{C}$ for 7 days. The cultured cells were then pelleted and lyophilized. The pellet was homogenized using a pre-chilled mortar and pestle. The pellet was extracted with 70\% ethanol using an ultra-sonication system (Vibra-Cell, Sonics \& Materials, Inc., Newtown, USA), followed by centrifugation at $5,000 \mathrm{rpm}$ at $4^{\circ} \mathrm{C}$ for $5 \mathrm{~min}$. The extracts were filtered through a $0.45-\mu \mathrm{m}$ membrane filter. The solvent was removed by a rotary evaporator (Buchi, Switzerland) and the resulting extract powder was dissolved in dimethyl sulfoxide (Sigma-Aldrich, USA) and stored at $-20^{\circ} \mathrm{C}$ until use.

\section{Reagents and Cell Culture}

B16F10 mouse melanoma cells were cultured in Roswell Park Memorial Institute 1640 medium (Biowest, Nuaillé, France) supplemented with $10 \%(\mathrm{v} / \mathrm{v})$ fetal bovine serum (Biowest), $100 \mathrm{U} / \mathrm{ml}$ penicillin, and $100 \mu \mathrm{g} / \mathrm{ml}$ streptomycin (Gibco, Grand Island, USA) in a humidified $5 \% \mathrm{CO}_{2}$ incubator at $37^{\circ} \mathrm{C}$. Normal human epidermal melanocytes (NHEM) were purchased from Thermo Fisher Scientific, Inc. (Waltham, USA). The cells were cultured in 254 Calcium Free Medium (Thermo Fisher Scientific, Inc.) supplemented with Human Melanocyte Growth Supplement-2 (Thermo Fisher Scientific Inc.) and $0.2 \mathrm{M}$ calcium chloride at $37^{\circ} \mathrm{C}$ in a humidified $5 \% \mathrm{CO}_{2}$ incubator.

\section{Determination of Photosynthetic Pigments Using High Performance Liquid Chromatography (HPLC)}

Microalgae were harvested by centrifugation at $6,000 \times g$ for $5 \mathrm{~min}$ and freeze-dried for further analysis. For the extraction of the biomass, the precisely-weighed $10 \mathrm{mg}$ of lyophilized samples were agitated and vortexed using $0.1 \mathrm{~mm}: 0.5 \mathrm{~mm}(1: 3, \mathrm{v} / \mathrm{v})$ zirconia beads (Biospec, Bartlesville, USA) with $1 \mathrm{ml}$ ethanol (Merck, Darmstadt, Germany) in a $1.5 \mathrm{ml}$ microcentrifuge tube. After being centrifuged at 10,000 $\times g$ for $1 \mathrm{~min}$, the supernatant phase was transferred into HPLC vials after filtering with $0.22 \mu \mathrm{m}$ polytetrafluoroethylene (PTFE) filters (Whatman, UK). The analysis of photosynthetic pigments was performed using the modified method of our previous reports $[15,16]$. Briefly, the photosynthetic pigment analysis conducted was by the Agilent 1260 Infinity Binary LC (Agilent Technologies, USA) system equipped with an autosampler and Waters Spherisorb S5 ODS1 $4.6 \times 250 \mathrm{~mm}, 5 \mu \mathrm{m}$ Cartridge Column (Waters Corporation, Milford, MA, USA) for separating samples. The injection volume was $20 \mu \mathrm{l}$, and analysis was conducted at $40^{\circ} \mathrm{C}$ for $25 \mathrm{~min}$. Then, $0.1 \mathrm{~mol} / 1$ Tris- $\mathrm{HCl}$ ( $\mathrm{pH} 8.0$ ), acetonitrile, methanol, and ethyl acetate were used for mobile phase, and gradient analysis was regulated by the following ratios; $14 \% 0.1 \mathrm{M}$ Tris $\mathrm{HCl}, 84 \%$ acetonitrile, 2\% methanol (0-15 $\mathrm{min}$ ), and 68\% methanol, 32\% ethyl acetate (15-19 $\mathrm{min}$ ), and $14 \%$ Tris $\mathrm{HCl}, 84 \%$ acetonitrile, $2 \%$ methanol $(19-25 \mathrm{~min})$ with $1.2 \mathrm{ml} / \mathrm{min}$ of flow rate. For identification and quantification of carotenoids in the extract, the peaks were identified by comparing the retention times with those 
of the corresponding standards. Carotenoids including $\beta$-carotene, lutein, neoxanthin, violaxanthin and others were purchased from Sigma-Aldrich (USA) and were used as standard. A standard curve was prepared for each sample and they were used for identification and quantification of carotenoid extracts.

\section{Cell Viability Assay}

Cell viability was measured using the water-soluble tetrazolium salt (WST-1) assay (EZ-Cytox Cell Viability Assay Kit; ITS-Bio, Korea). Absorbance was measured at $450 \mathrm{~nm}$ using an iMark microplate reader (Bio-Rad, USA)

\section{Determination of Melanin Content}

To measure melanin production, the cells were treated with different concentrations of CE extract in the presence or absence of stimulating agents (100 nM $\alpha$-MSH or arbutin) for $48 \mathrm{~h}$. After harvesting and centrifugation for $5 \mathrm{~min}$ at $12,000 \mathrm{rpm}$ at $4{ }^{\circ} \mathrm{C}$, the cell pellets were photographed and then solubilized in $1 \mathrm{~N} \mathrm{NaOH}$ lysis buffer at $60^{\circ} \mathrm{C}$ for $1 \mathrm{~h}$. Melanin content was measured by comparing the absorbance at $450 \mathrm{~nm}$ measured using an iMark microplate reader (Bio-Rad). Total protein was measured using the Pierce BCA protein assay kit (Rockford, USA). Relative melanin contents were calculated from a standard curve using the total protein concentration.

\section{Measurement of Tyrosinase Activity}

To measure tyrosinase activity in a cell-free system, $1 \mathrm{mM} \mathrm{L}$ DOPA solution was added to a 96-well plate and reacted with various concentrations of $\mathrm{CE}$ extract in $0.1 \mathrm{M}$ sodium phosphate buffer (pH 6.8) and $100 \mathrm{U} / \mathrm{ml}$ mushroom tyrosinase solution. After incubation for $5 \mathrm{~min}$ at $37^{\circ} \mathrm{C}$, the production of dopachrome was determined by monitoring the change in absorbance at $450 \mathrm{~nm}$ using an iMark microplate reader. To measure cellular tyrosinase activity, the supernatant was mixed with $10 \mathrm{mM}$ L-DOPA solution and incubated for $60 \mathrm{~min}$ at $37^{\circ} \mathrm{C}$. The relative tyrosinase activity was determined by measuring the amount of dopachrome formed using ${ }_{\mathrm{L}}$-DOPA as the substrate and compared to the control. Dopachrome formation was measured by determining the absorbance at $450 \mathrm{~nm}$ using an iMark microplate reader (Bio-Rad). The relative tyrosinase activity was calculated from a standard curve using the total protein concentration.

\section{RNA Analysis.}

Total RNAs were isolated using the TRIzol reagent (Invitrogen, USA). cDNA was synthesized using $1 \mu \mathrm{g}$ of total RNA with the Moloney Murine Leukemia Virus reverse transcriptase according to the manufacturer's protocol (Invitrogen). Quantitative realtime PCR (qRT-PCR) was performed using the StepOnePlus RealTime PCR system (Thermo Fisher Scientific) and analyzed using the Relative Quantification Study software (Thermo Fisher Scientific). Each reaction was performed using a HOT FIREPol EvaGreen qRCR Mis Plus (Solis BioDyne, Tartu, Estonia) in triplicate for three independent runs. Relative quantification of gene expression was calculated as the fold-change according to the $2{ }^{-\Delta \mathrm{Ct}}$ method and normalized against an endogenous internal control ( $\beta$-actin). The primer sequences were as follows: Mitf forward, 5'-AGAAGCTGGAGCATGCGAACC-3' and Mitf reverse, 5'-GTTCCTGGCTGCAGTTCTCAAG-3'; Tyrosinase forward, 5'ACACACTGGAAGGATTTGCC- $3^{\prime}$ and Tyrosinase reverse, $5^{\prime}$ GAGCGGTATGAAAGGAACCA-3'; Tyrp-1 forward, 5'- GATGTC TGCACTAGTGACTTG-3' and Tyrp-1 reverse, 5' - CCTGATTGG TCCACCCTCAG-3'; Tyrp-2 forward, 5'-CGTGCTGAACAAGGA ATGCT-3' and Tyrp-2 reverse, 5'-GCATGTCCGGTTGAAGAAT-3'; $\beta$-actin forward, $5^{\prime}$-GTATGGAATCCTGTGGCATC $3^{\prime}$ and $\beta$-actin reverse, 5'-AAGCACTTGCGGTGCACGAT-3'.

\section{Western Blot Analysis}

Total cell lysates were prepared, after which protein extracts were subjected to sodium dodecyl sulfate-polyacrylamide gel electrophoresis and transferred to nitrocellulose membranes. The membranes were incubated overnight at $4^{\circ} \mathrm{C}$ with primary antibodies against Mitf, Tyrosinase, Tyrp-1, Tyrp-2, p38 (Santa Cruz Biotechnology, USA), p-PKA (Thr198), CREB, p-CREB (Ser133), ERK, p-ERK (Thr202/Tyr204), p-p38 (Thr180/Tyr182), JNK, p-JNK (Thr183/Tyr185) (Cell Signaling Technology, USA), and PKA (Abcam, UK); with suitable horseradish peroxidaseconjugated secondary antibodies for detection. Each protein was detected using ECL reagent (Bio-Rad) and visualized using the ChemiDoc Touch Imaging System (Bio-Rad). Relative protein expression was normalized against an endogenous internal control, $\beta$-actin.

In Vitro 3D-Reconstructed Human Pigmented Epidermis Model A three-dimensional reconstructed human pigmented epidermis (3D-RHPE) model composed of normal human-derived epidermal keratinocytes (NHEKs) and melanocytes (NHEMs) was prepared. Briefly, type I collagen matrix (Thermo Fisher Scientific) was coated onto a polycarbonate insert (Millipore, USA). NHEKs and NHEMs were incubated on the inserts in proliferation medium for 3 days at $37^{\circ} \mathrm{C}$ with $5 \% \mathrm{CO}_{2}$. All media were removed from the inside of the insert and replaced with fresh medium containing $100 \mathrm{nM} \alpha-\mathrm{MSH}$ in the presence or absence of either CE extract or arbutin on the outside of the insert. The conditioned medium was replaced inside the insert every 2 days for 14 days. The 3D-RHPE models were fixed with $4 \%$ paraformaldehyde and embedded in wax.

\section{Hematoxylin and Eosin (H\&E) and Fontana-Masson Staining Assays}

For histological analysis of the 3D-RHPE model, the tissue was stained with hematoxylin and eosin (H\&E). The tissue was sectioned (7- $\mu \mathrm{m}$ thickness) and de-paraffinized by washing with xylene three times for $1 \mathrm{~min}$ and 100\%,95\%,90\%, 80\%, and 70\% ethyl alcohol for $30 \mathrm{~s}$ each. The sections were stained with Mayer's Hematoxylin (Merck, Darmstadt, Germany) for $60 \mathrm{sec}$ and washed with tap water for $5 \mathrm{~min}$. Sections were counterstained by 
rinsing with Eosin Y-solution (Merck) for 4 min, while dehydration was performed by sequentially washing with $95 \%$ ethyl alcohol, $100 \%$ ethyl alcohol, and xylene for $30 \mathrm{~s}$ each. Mounting solution (Thermo Fisher Scientific) was added to the slides, which were covered prior to examination by light microscopy using an Olympus CKX 41 microscope (Japan). To quantify melanin in the 3D-RHPE model, Fontana-Masson staining was performed on the formalin-fixed, paraffin-embedded tissue. The tissue section was treated with $2.5 \%$ aqueous silver nitrate solution for $10 \mathrm{~min}, 0.2 \%$ aqueous gold chloride for $1 \mathrm{~min}$, and $5 \%$ aqueous sodium thiosulfate for $5 \mathrm{~min}$. The number of pixels representing FontanaMasson staining were normalized to the total amount of epidermal counterstain. The values were expressed as the mean value \pm SD. Statistical analysis was performed using Student's $t$-test and the significance level was set to $p<0.05$.

A

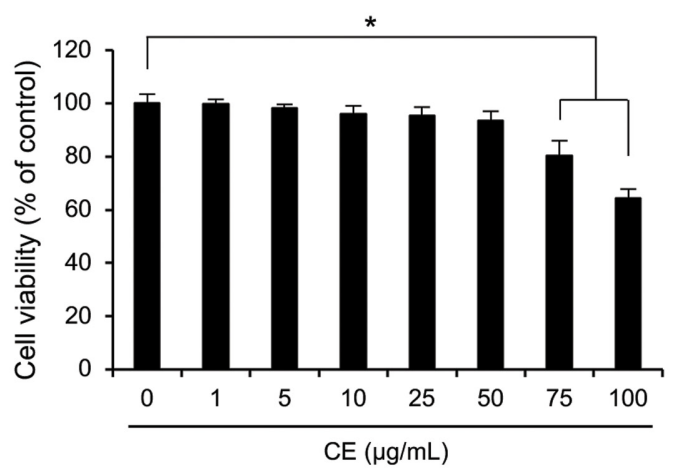

C

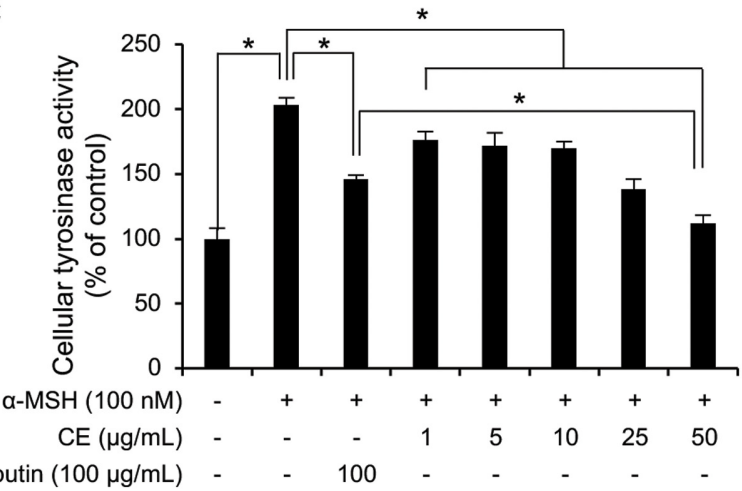

\section{Statistical Analysis}

All data are presented as mean \pm SD and were analyzed using the Student's $t$-test or one-way ANOVA followed by Tukey's test. All analyses were performed in triplicates, and $p$-values $<0.05$ were considered significant.

\section{Results}

\section{CE Extract Reduces Melanin Content in B16F10 Cells}

To determine whether CE extract has cytotoxic effects, B16F10 cells treated with different concentrations (1$100 \mu \mathrm{g} / \mathrm{ml}$ ) of the extract for $48 \mathrm{~h}$ were assayed for cell viability. As shown in Fig. 1A, treatment with the extract at concentrations of up to $50 \mu \mathrm{g} / \mathrm{ml}$ did not affect viability; however, higher concentrations significantly reduced cell
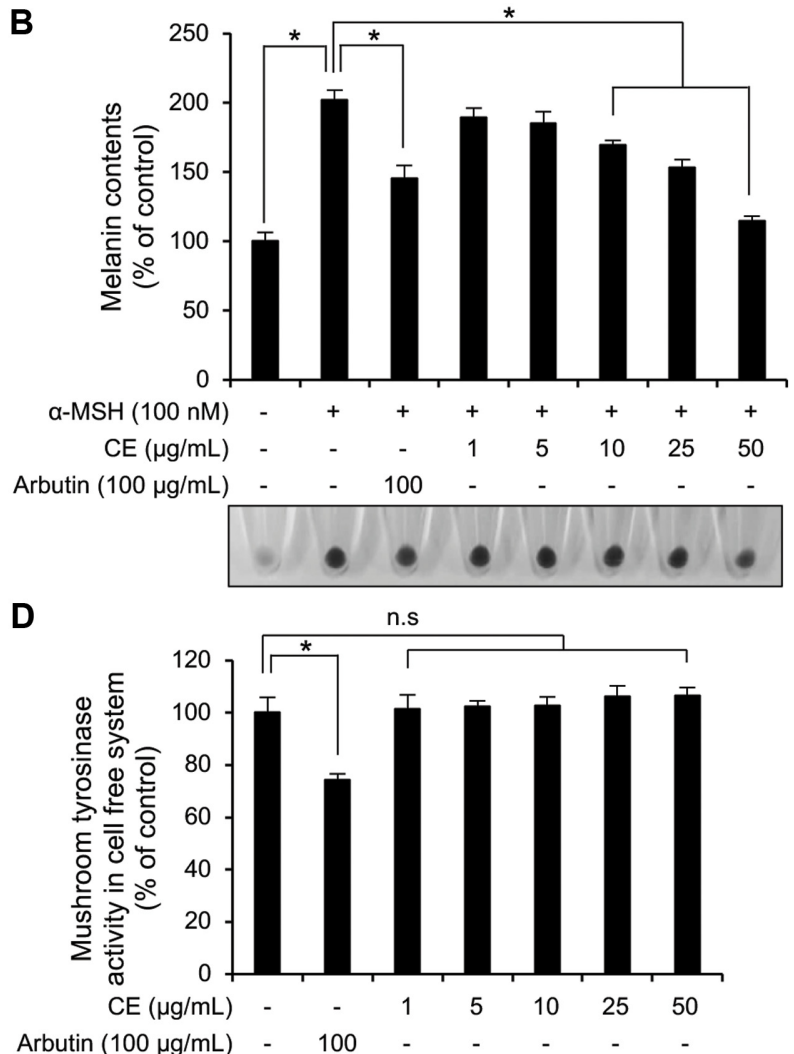

Fig. 1. Effect of CE extract on melanogenesis in B16F10 cells.

(A) B16F10 cells were treated with different concentrations of the CE extract (1-100 $\mu \mathrm{g} / \mathrm{ml})$ for $48 \mathrm{~h}$ and cell viability was determined through WST-1 assay. (B) B16F10 cells were co-cultured with $\alpha-\mathrm{MSH}(100 \mathrm{nM})$ and $\mathrm{CE}$ extract $(1-50 \mu \mathrm{g} / \mathrm{ml})$ for $48 \mathrm{~h}$ and were observed for the accumulation of melanin through the pigmentation of cell pellets. Melanin content was determined by measuring the amount of dopachrome formed. Arbutin was applied used as a positive control. (C) B16F10 cells were co-cultured with $\alpha-\mathrm{MSH}(100 \mathrm{nM})$ and CE (1-50 $\mu \mathrm{g} / \mathrm{ml})$ for $48 \mathrm{~h}$ and cellular tyrosinase activity was determined by measuring dopachrome formation from the L-DOPA substrate. (D) Mushroom tyrosinase activity was measured by measuring the formed dopachrome, which directly reacted with the L-DOPA substrate. The results are expressed as percent relative to the control. The data are presented as the mean \pm SD of three independent experiments. Significance was determined using a one-way ANOVA followed by Tukey's test. Values of ${ }^{*} p<0.05$ were considered to be statistically significant. n.s., non-significant. 
viability compared to the control. Next, to determine whether non-cytotoxic concentrations of extracts have a regulatory effect on melanogenesis, the cells were cotreated with $\alpha-\mathrm{MSH}$ and the extract for $48 \mathrm{~h}$ and then analyzed for melanin content; arbutin was used as a positive control. As shown in Fig. 1B, treatment with the extract had significant concentration-dependent antipigmentation effects in $\alpha$-MSH-stimulated B16F10 cells as revealed by the melanin content assay. Notably, the inhibitory ratio on melanin content of the extract with a concentration of $50 \mu \mathrm{g} / \mathrm{ml}$ was higher than that of the positive control.

We further examined whether the inhibitory effect of the extract on melanin synthesis was mediated by regulating cellular tyrosinase activity. B16F10 cells were cultured under the same experimental conditions and cellular tyrosinase activity was measured by determining L-DOPA oxidation values. As expected, treatment with the extract resulted in significant concentration-dependent inhibition of tyrosinase activity (Fig. 1C). Furthermore, we determined whether the extract directly inhibited tyrosinase activity in an in vitro mushroom tyrosinase assay. The results showed that mushroom tyrosinase activity was not affected by the extract at any concentration tested (Fig. 1D), indicating that the extract does not directly inhibit the catalytic activity of tyrosinase.

\section{CE Extract Downregulates the Expression of Melanogenesis-} Related Genes in B16F10 Cells

The results in Fig. 1 show the inhibitory effect of the CE extract on tyrosinase activity in vitro. We further evaluated how the extract negatively regulates cellular tyrosinase activity and the mechanisms involved in the antipigmentation effect of the extract. We first analyzed the expression levels of tyrosinase, tyrosinase-related protein 1 (Tyrp-1), and tyrosinase-related protein 2 (Tyrp-2) by immunoblotting. As shown in Fig. 2A, tyrosinase, Tyrp-1, and Tyrp-2 protein expression levels were greater in $\alpha$ MSH-treated B16F10 cells than in control cells; however, $\mathrm{CE}$ extract markedly decreased the expression levels of these proteins in a dose-dependent manner. We further analyzed the mRNA levels of Tyrosinase, Tyrp-1, and Tyrp-2 by qRT-PCR and found that treatment with the extract significantly decreased mRNA expression of these genes in a dose-dependent manner (Figs. 2B-2D). Because Tyrosinase, Tyrp-1, and Tyrp-2 are transcribed by Mitf, we examined whether the CE extract also regulates the levels of Mitf. As shown in Figs. $2 \mathrm{E}$ and $2 \mathrm{~F}$, the extract significantly downregulated both the protein and mRNA levels of Mitf in a dose-dependent manner. Taken together, these results suggest that the anti-pigmentation effect of the extract is attributed to downregulation of Mitf expression in cells.

\section{CE Extract Downregulates Phosphorylation of PKA and ERK Proteins}

Mitf expression is also known to be regulated by the cAMP-mediated PKA/CREB signaling pathway [10]. We therefore investigated the effects of CE extract on the PKA/ CREB signaling pathway. The results showed that the protein levels of PKA and CREB were not affected; however, the phosphorylation levels of PKA and CREB in $\alpha$-MSH-stimulated B16F10 cells were largely reduced by treatment with the extract in a dose-dependent manner (Figs. 3A and 3B). In melanogenesis, phosphorylation of CREB is also induced by ERK in a PKA-independent manner $[9,17]$. Therefore, we further investigated whether the extract regulates ERK activation/phosphorylation in B16F10 cells. Treatment with the extract reduced $\alpha-\mathrm{MSH}-$ induced ERK phosphorylation; however, the phosphorylation levels of p38 and JNK proteins were not affected by the extracts (Fig. 3C). These results suggest that the CE extract inhibits Mitf expression by suppressing both the PKA/ CREB and ERK/CREB pathways.

\section{Inhibitory Effect of CE Extract on Melanogenesis in 3D- RHPE Model}

Cell viability assays revealed that extract concentrations below $5 \mu \mathrm{g} / \mathrm{ml}$ had minimal cytotoxic effects on NHEM cells (Fig. 4A). Consistent with the anti-pigmentation effects on mouse B16F10 cells, the non-cytotoxic concentration of the extract significantly inhibited melanin production induced by $\alpha-\mathrm{MSH}$ in NHEM cells (Fig. 4B). In 3D-RHPE model experiments, we found that pigmentation was markedly decreased by treatment with CE extract compared to cells treated with phosphate-buffered saline and $\alpha-\mathrm{MSH}$ (Fig. 4C). Additionally, after staining with Fontana-Masson, relative hypopigmentation was observed in the 3D-RHPE model compared to after treatment with phosphate-buffered saline and $\alpha-\mathrm{MSH}$ (Fig. 4D); pigmentation levels were quantified and are shown in Fig. 4E. These results indicate that $\mathrm{CE}$ extract is an effective inhibitor of hyperpigmentation.

\section{Carotenoid Composition in CE Extract}

It has been reported that the abnormal and overproduction of melanin is tightly linked to UV-induced intracellular ROS production in melanocytes [18, 19]. Numerous studies have proven that carotenoids could exert as efficient ROS scavengers and have a protective function against ultraviolet 
A

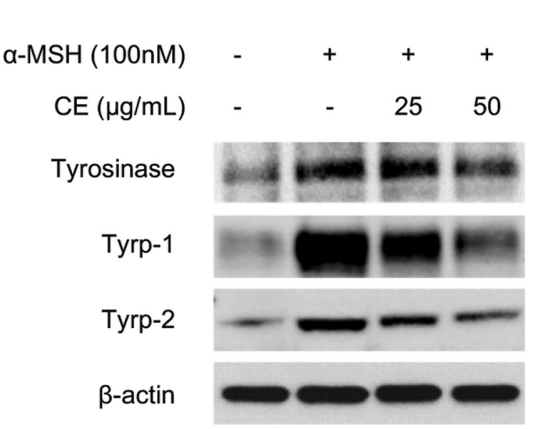

B

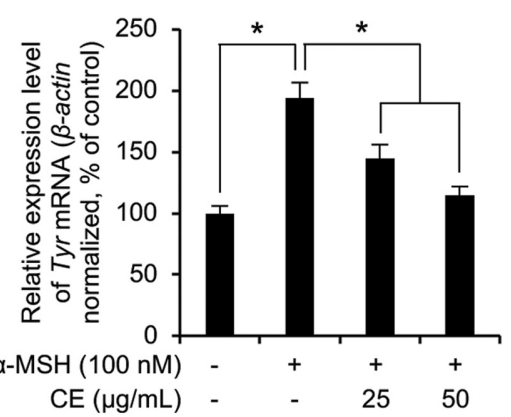

E

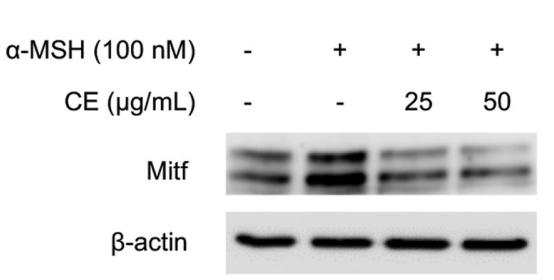

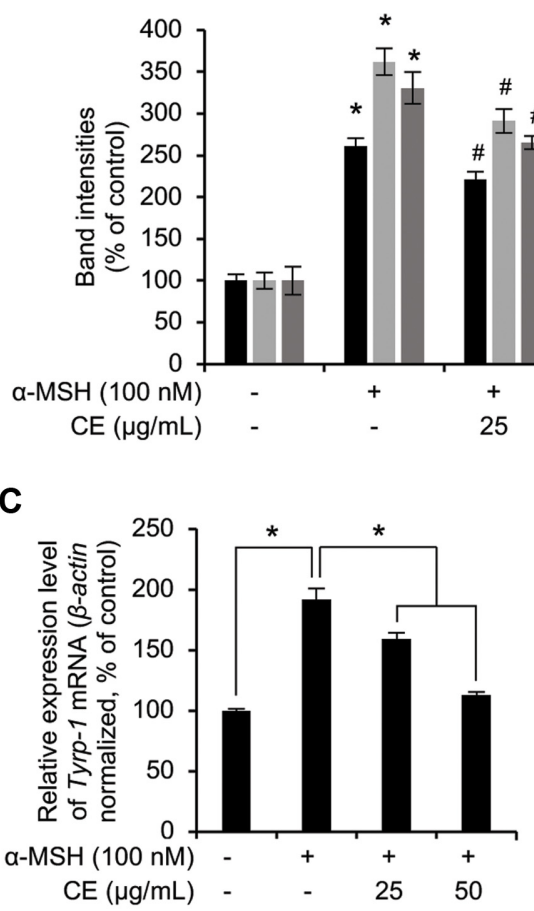

D

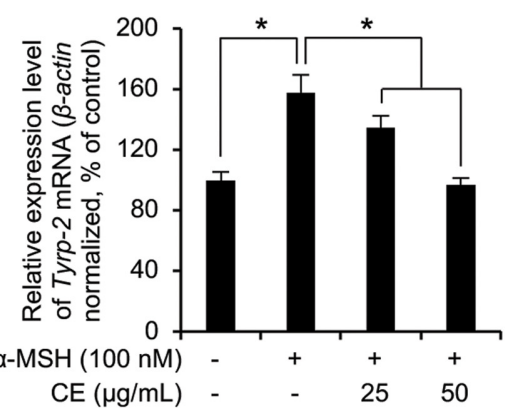

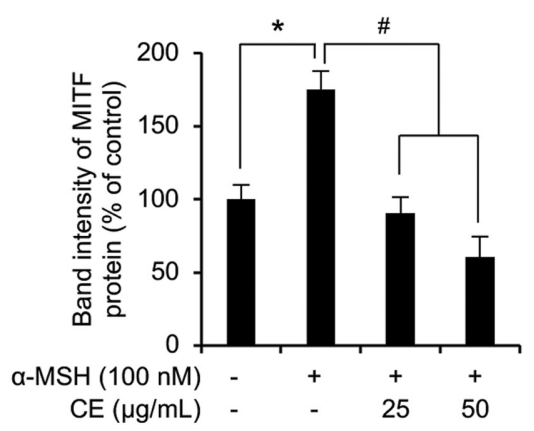

$\mathbf{F}$

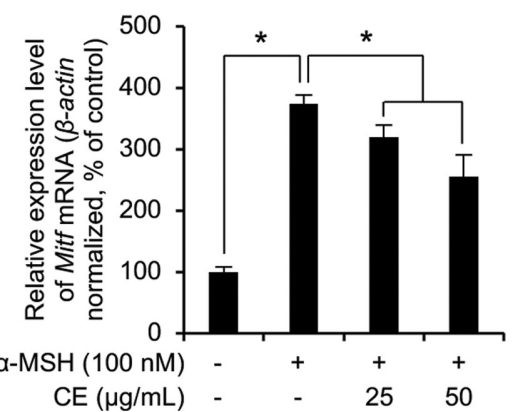

Fig. 2. Effect of CE extract on the expression of melanogenesis-related proteins in B16F10 cells.

(A) The expression levels of Tyrosinase, Tyrp-1 and Tyrp-2 were downregulated by CE extract in B16F10 cells. Cells were co-treated with $\alpha-\mathrm{MSH}$ $(100 \mathrm{nM})$ and CE extract $(25$ and $50 \mu \mathrm{g} / \mathrm{ml})$ for $24 \mathrm{~h}$. Cells were lysed and the indicated protein levels were examined using immunoblotting with their specific antibodies. Quantification of Tyrosinase, Tyrp-1 and Tyrp-2 blots was analyzed using the Image-J program and normalized to $\beta$-actin blots. The expression levels of Tyrosinase (Try) (B), Tyrp-1 (C) and Tyrp-2 (D) mRNAs were measured using qRT-PCR with their specific primers. (E and F) CE extract downregulates the expression levels of Mitf. Cells were co-treated with $\alpha-\mathrm{MSH}$ and CE extract, and immunoblotting and qRTPCR were conducted to examine the protein and mRNA levels of Mitf using the specific antibody and primer, respectively. The results were normalized against $\beta$-actin. The data are presented as the mean \pm SD of three independent experiments. Quantification of Mitf blot were analyzed using the Image-J program and normalized to $\beta$-actin blots. Significance was determined using a one-way ANOVA followed by Tukey's test. Values of ${ }^{*} p<0.05$ were considered to be statistically significant.

(UV)-induced ROS production and hyperpigmentation in melanocytes [18, 19]. Therefore, we determined the carotenoid composition of the extracts using HPLC analysis and found 6 different carotenoid peaks including neoxanthin, violaxanthin, lutein, $\beta$-carotene, chlorophyll-b and chlorophyll-a (Fig. 5A). Further analysis determined each carotenoid composition ratio compared to total pigments (Fig. 5B) and pigment content compared to biomass (Fig. 5C), including neoxanthin $(2.482 \mathrm{mg} / \mathrm{g} ; 5.909 \%$ of total composition), violaxanthin $(1.200 \mathrm{mg} / \mathrm{g} ; 2.858 \%)$, lutein $(3.293 \mathrm{mg} / \mathrm{g} ; 7.841 \%), \beta$-carotene $(5.956 \mathrm{mg} / \mathrm{g} ; 14.182 \%)$, chlorophyll-b $\quad(11.500 \mathrm{mg} / \mathrm{g} ; \quad 27.382 \%)$, chlorophyll-a 
A

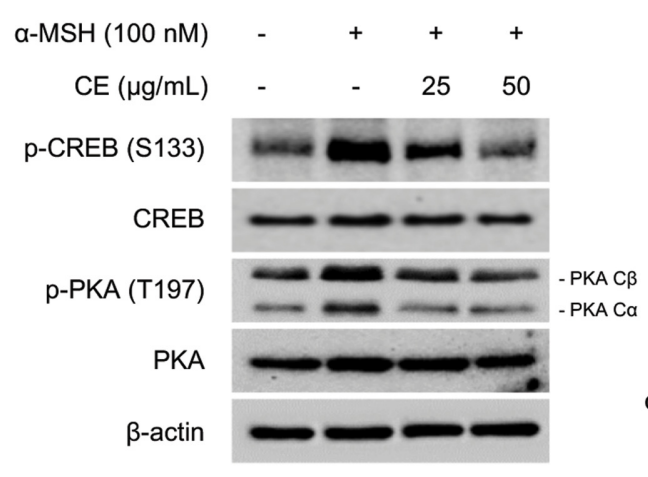

B
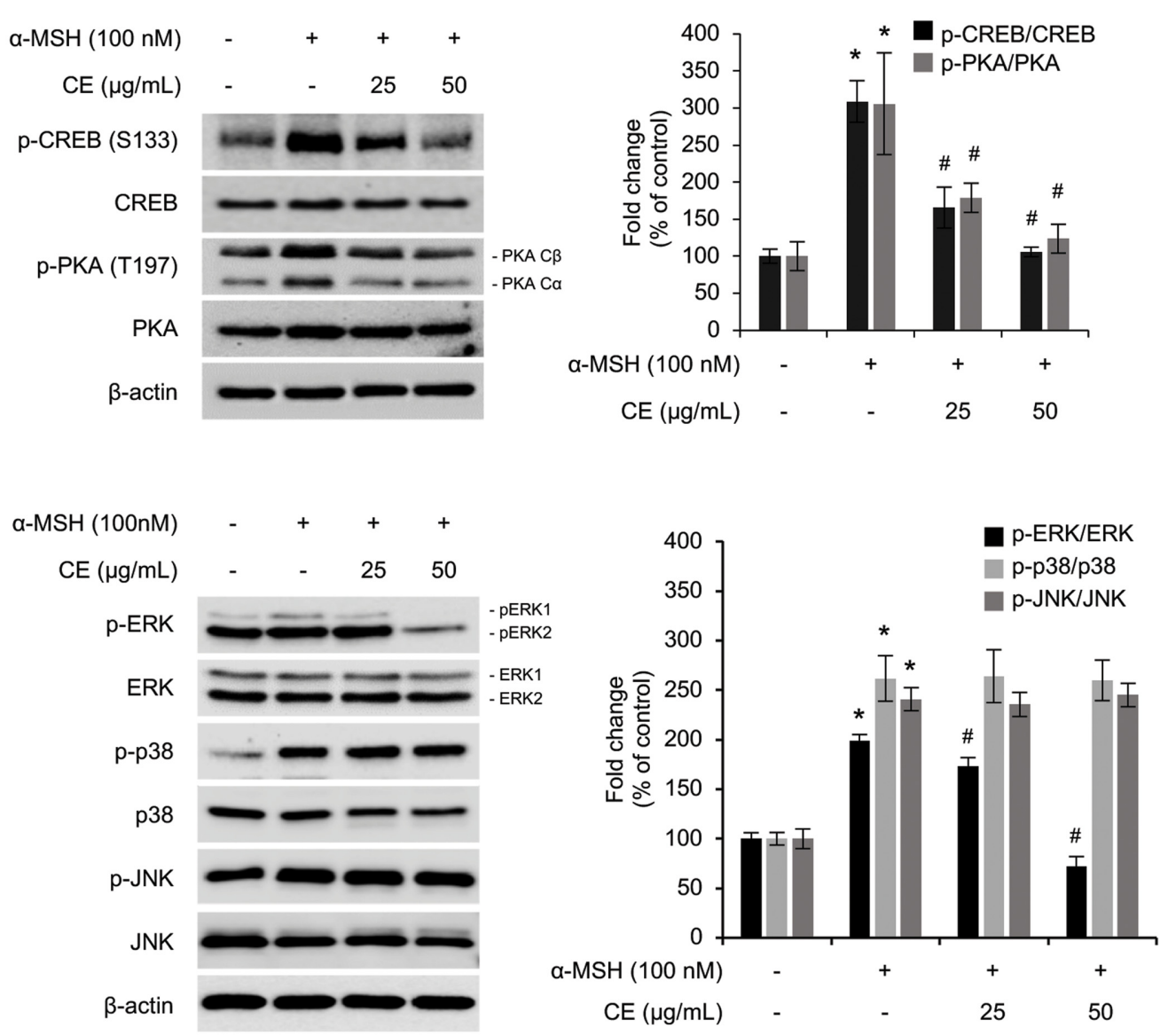

Fig. 3. Effect of CE extract on phosphorylation of PKA and ERK in B16F10 cells.

B16F10 cells were co-cultured with $\alpha-\mathrm{MSH}(100 \mathrm{nM})$ and CE extract $(25,50 \mu \mathrm{g} / \mathrm{ml})$. Changes in the levels of p-PKA, p-CREB (A and B), and pMAPKs including p-ERK, p-p38, and p-JNK (C) were examined by western blot analysis. The results were normalized against $\beta$-actin. (B) Quantification of p-CREB, p-PKA, p-ERK, p-p38, and p-JNK blots was analyzed using the Image-J program and normalized to the total protein CREB, PKA, ERK, p38 and JNK blots, respectively. The data are presented as the mean $\pm \mathrm{SD}$ of three independent experiments. Significance was determined using a one-way ANOVA followed by Tukey's test. ${ }^{*} p<0.05$ compared with the vehicle treated group. \#p $<0.05$ compared with $\alpha$ MSH treated group. Values of $p<0.05$ were considered to be statistically significant.

$(14.381 \mathrm{mg} / \mathrm{g} ; 34.242 \%)$ and others $(3.186 \mathrm{mg} / \mathrm{g} ; 7.586 \%)$ (Figs. 5B and 5C).

\section{Discussion}

When human skin is exposed to UV radiation, hyperpigmentation and intracellular ROS production are accumulated and induce various types of cellular damage [20]. Carotenoids are known to be very efficient physical and chemical quenchers of singlet oxygen, as well as potent scavengers of other reactive oxygen species (ROS) [21]. Therefore, carotenoids such as fucoxanthin, astaxanthin, and $\beta$-carotene have proven to offer protection against ultraviolet (UV)-induced skin damage such as hyperpigmentation and oxidative damage in skin cells [18,
19]. Notably, fucoxanthin exerts its effect by suppressing prostaglandin E2 synthesis and melanogenic stimulant receptors [18]. Astaxanthin and $\beta$-carotene exhibit a photoprotective effect by expressing oxidative stressresponsible enzymes, such as heme oxygenase-1 [19]. Moreover, it has been known that phenolic compounds act as antioxidants by preventing radical formation [22]. Polyphenols including flavonoids, lignins, and phenolic acids are known to exhibit a broad spectrum of chemical and biological antioxidant activities [23]. However, the effect of $C$. reinhardtii containing such biologically active substances on melanogenesis has not been established. Therefore, our study investigated the anti-melanogenetic effect of C. reinhardtii extracts in B16F10 cells and normal human epidermal melanocytes. Using HPLC analysis, we 
A

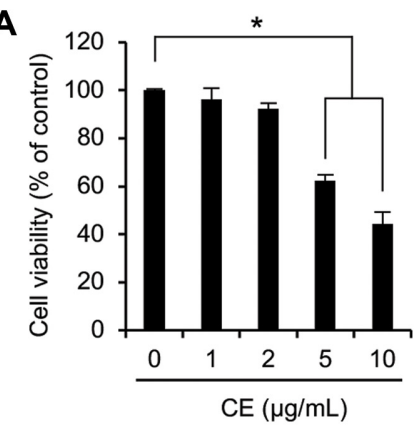

B

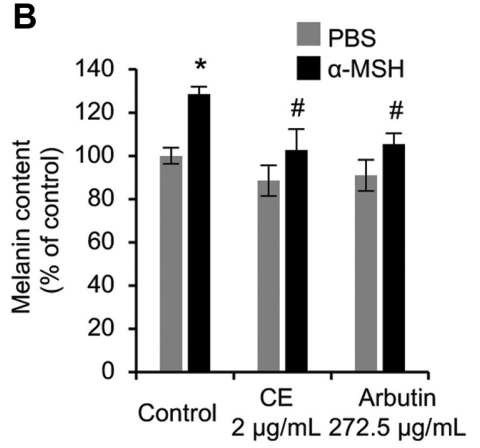

C

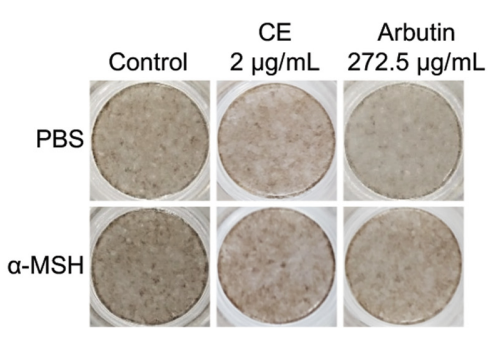

E

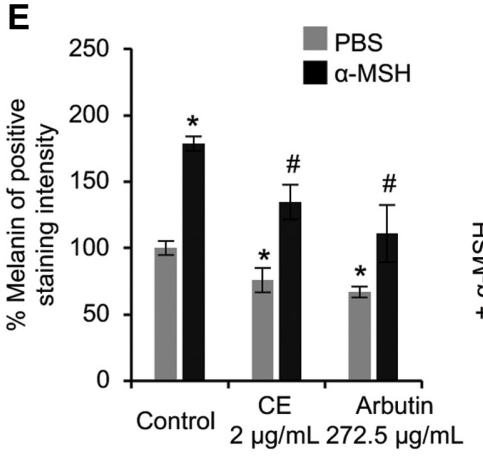

D
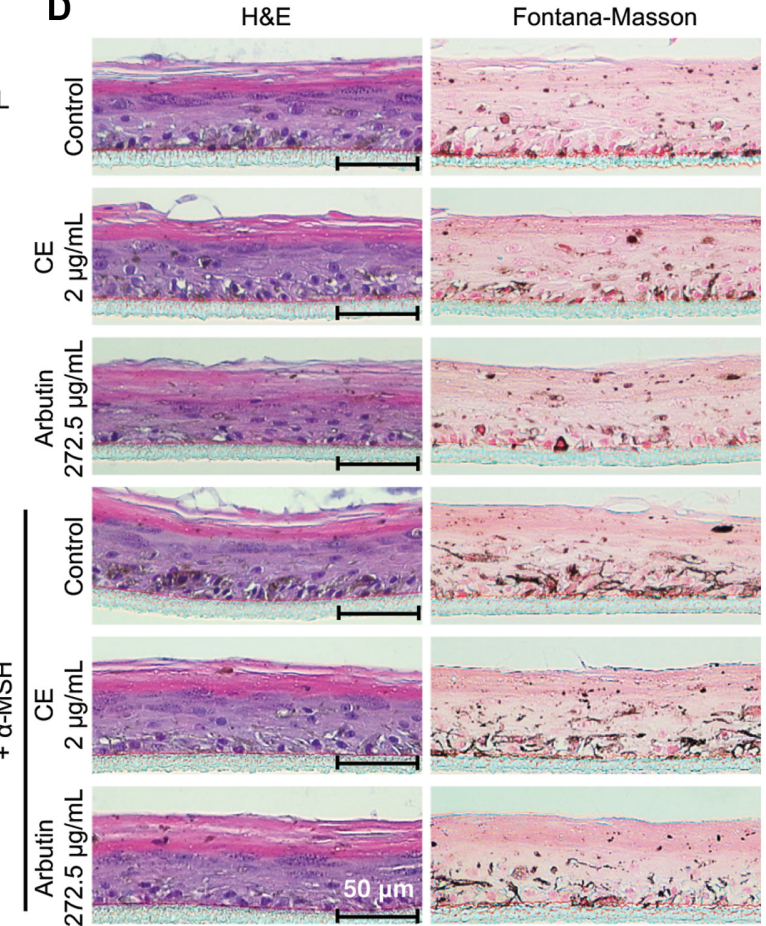

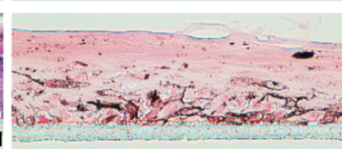

6

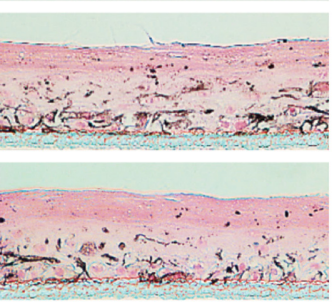

Fig. 4. CE extract decreased the level of melanin in 3D-reconstructed human pigmented epidermis (3D-RHPE) model.

(A) Cell viability and (B) melanin content was determined in NHEM cells treated with CE (1-10 $\mu \mathrm{g} / \mathrm{ml})$ or arbutin $(272.5 \mu \mathrm{g} / \mathrm{ml})$ in the presence or absence of $\alpha$-MSH $(100 \mathrm{nM})$. Arbutin was applied as a positive control. (C-E) 3D-RHPE models were exposed to CE extract ( $2 \mu \mathrm{g} / \mathrm{ml})$ or arbutin $(272.5 \mu \mathrm{g} / \mathrm{ml})$ every 2 days for 14 days in the presence or absence of $\alpha-\mathrm{MSH}(50 \mathrm{nM})$. (C) Images were obtained using a microscope. (D) Pigmentation was observed upon Fontana-Masson (FM) staining. (E) Quantification of Fontana-Masson staining was analyzed using the Image-J program. The data are presented as the mean \pm SD of three independent experiments. Significance was determined using a one-way ANOVA followed by Tukey's test. * $p<0.05$ compared with the vehicle treated group. $\# p<0.05$ compared with $\alpha$-MSH treated group. Values of $p<0.05$ were considered to be statistically significant.

further found that the extract had several carotenoids, including neoxanthin, violaxanthin, lutein, $\beta$-carotene, and others, with different composition levels. These data had a limitation for additional interpretation because further studies are needed to elucidate which of the carotenoids in the extract specifically regulate melanogenesis in the melanocytes. Although the specific substances and mechanisms remain to be fully elucidated, our data indicate that the extract is a potential natural source of antipigmentation agents that could be used in the pharmaceutical and cosmetic industries.

Inhibiting tyrosinase activity is the main strategy for anti-pigmentation and skin whitening as this enzyme catalyzes two important reactions that lead to the production of melanin [24]. Our data showed that the CE extract significantly decreased the cellular melanin content in a dose-dependent manner; however, this result was not due to a direct inhibition of tyrosinase activity, indicating that the CE extract may exert its effects through different inhibition pathways that are not based on the catalytic activity of tyrosinase. There are two effective approaches in exerting anti-pigmentation or skin whitening effects, i.e., targeting the activities of tyrosinase and Mitf [25]. We found that the mRNA expression of tyrosinase was significantly repressed by the CE extract. Moreover, the transcription of other genes essential for melanin biosynthesis, such as Tyrp-1 and Tyrp-2, was also downregulated. Because tyrosinase, Tyrp-1, and Tyrp-2 share Mitf as a common transcription factor, one possible explanation may be that the extract has an inhibitory effect on the protein function [25]. It has been known that Mitf activity is regulated by its upstream regulators operating on transcriptional and posttranslational levels [26]. Especially, double phosphorylation on Ser-73 and Ser-409 leads to impairment of Mitf activity though proteasome-mediated protein degradation [26]. We therefore further investigated the effect of $\mathrm{CE}$ extract on regulation of Mitf function by analyzing the expression levels of Mitf mRNA and protein. Immunoblotting and 
A

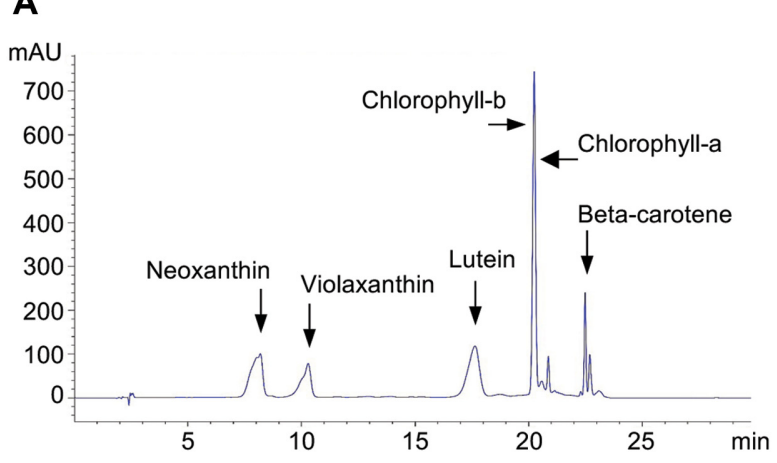

B

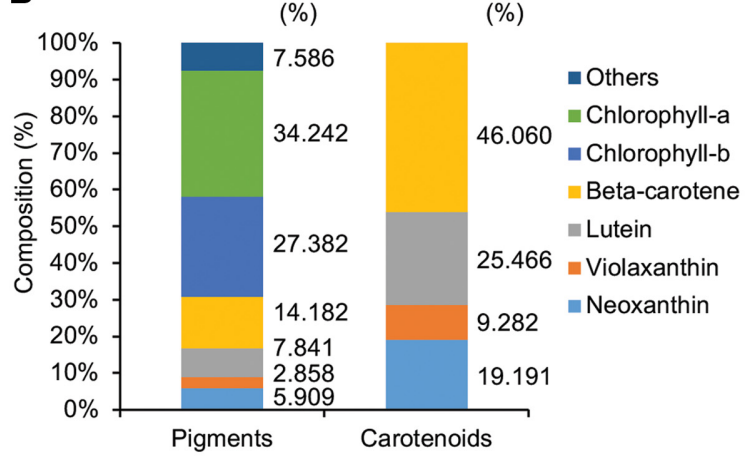

C

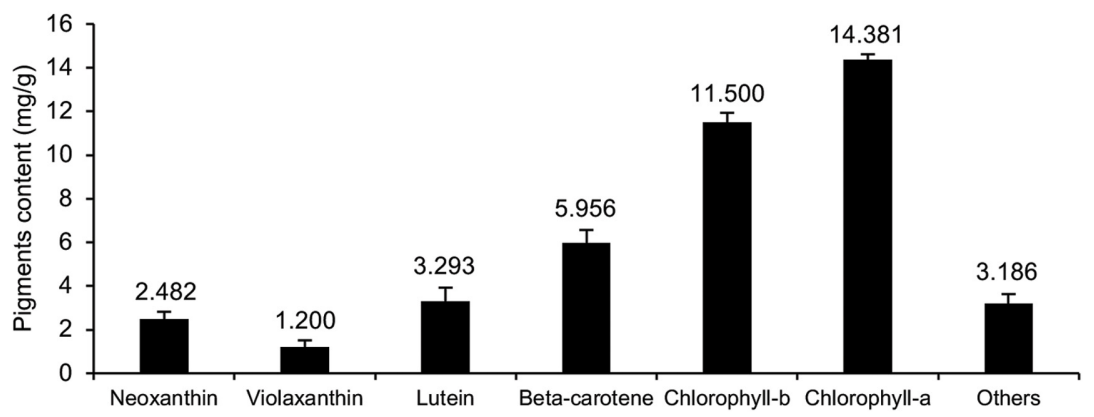

Fig. 5. Carotenoid composition and concentration in CE extract.

(A) HPLC chromatogram at $445 \mathrm{~nm}$ of CE extract. (B and C) Composition and concentration of individual carotenoids containing neoxanthin, violaxanthin, lutein, $\beta$-carotene, and others in CE extract. Percentage of peak area in the HPLC chromatogram at 445 nm. Identification and quantification were achieved by the individual standard carotenoids.

qRT-PCR assays showed that treatment with CE extract decreased the expression of both Mitf mRNA and protein, indicating that CE extract-mediated anti-pigmentation effect was involved in the transcriptionally downregulated Mitf expression in B16F10 cells. Other studies have revealed that inhibition or regulation of MITF as well as tyrosinase could be an important strategy for skin whitening [27], and the evaluation of cellular MITF expression is able to identify whitening substances [27]. Therefore, our data suggest that CE extract could be a novel agent with whitening potency. However, the exact anti-pigmentation mechanisms of CE extract and its clinical efficiency should be investigated in future studies.

Our findings allowed us to investigate the mechanisms responsible for Mitf downregulation in B16F10 cells treated with the extract. The transcriptional regulation of Mitf is affected by several transcription factors and their partners associated with diverse signaling pathways [26]. In melanogenesis, one specialized factor, $\alpha-\mathrm{MSH}$, is secreted from keratinocytes irradiated with UV rays and has a pivotal role in melanin synthesis through stimulation of Mitf transcription [26]. Our data showed that the treatment with CE extract significantly decreased intracellular melanin concentration in B16F10 cells stimulated by $\alpha-\mathrm{MSH}$; therefore, we hypothesized that the anti-pigmentation effect of the extract would be mediated by inhibition of the signaling pathway responsible for $\alpha$-MSH-induced Mitf expression. Studies has revealed that $\alpha-\mathrm{MSH}$ directly binds to its receptor $\mathrm{MC1R}$, and activates the enzyme adenylate cyclase, increasing cAMP levels, activating PKA, and CREB phosphorylation/activation in melanocytes [28]. Phosphorylated CREB promotes its binding to the Mitf promoter [10]. As expected, our data clearly showed that the treatment with the extract inhibited the phosphorylation of PKA and CREB in $\alpha-\mathrm{MSH}$-stimulated B16F10 cells, indicating that $\mathrm{CE}$ extract-mediated Mitf downregulation was mediated by inhibition of $\alpha-\mathrm{MSH}$-induced PKA/CREB pathway. Consistent with our data, several agents including bisabolangelone and $\alpha$-viniferin, and the specific PKA inhibitor, H89, function as anti-pigmentation agents through PKA/CREB inhibition in melanocytes [29-31]. Future studies will be focused on the exact mechanisms and molecular target of CE extract-mediated PKA/CREB pathway inhibition in melanocytes. 
It is well established that Mitf expression can be also regulated by mitogen-activated protein kinases (MAPKs), which are a family of conserved protein serine-threonine kinases that include ERK, p38, and JNK [32, 33]. Indeed, Studies have shown that MAPKs are major intracellular signaling molecules critical to pigmentation and are known to induce melanin synthesis $[32,33]$. After activating MC1R by $\alpha-\mathrm{MSH}$ in melanocytes, ERK phosphorylation can increase the transcriptional activity of Mitf [33]. Other reports have also shown that p38 phosphorylation increases Mitf expression levels, whereas the phosphorylation of JNK downregulates melanin synthesis [34, 35]. Our results in B16F10 cells showed that the phosphorylation of ERK was significantly decreased by the CE extract, whereas phosphorylation of either p38 or JNK was not affected, indicating that the reduction in the anti-pigmentation effect of the extract is mediated by the inhibition of PKA and ERK phosphorylation. Consistent with our results, upon UV irradiation, stimulation of melanogenesis is associated with the phosphorylation/activation of ERK, but not JNK and p38, in human melanocytes [36]. Moreover, it has been reported that cAMP-induced melanogenesis is linked to the activation of ERK pathway in B16 melanoma cells [37]. On the contrary, it has been reported that activation of the ERK pathway leads to the inhibition of melanogenesis rather than activation of it; however, these results were shown in melanoma cells stimulated with a spingosin-1-phosphate, not an $\alpha-\mathrm{MSH}$ [38]. Further validation of the ERK pathway in $\alpha$-MSH-induced melanogenesis with additional in-depth studies in the future is required to better understand the mechanisms of this anti-pigmentation effect.

The 3D tissues exhibited morphological equivalence with human skin and represented in vivo-like histological morphology, consisting of a multi-layered, stratified, and pigmented epidermis [39]. Also, for translational reasons, there is a preference for using a 3D human skin equivalent rather than 2D-based experiments. Recently, the OECD has adopted the in vitro skin corrosion and irritation tests by using the reconstructed 3D human skin models [40, 41]. To confirm our results in the mouse B16F10 cell culture system, we also performed tests using normal human epidermal melanocytes (NHEM) and 3D-reconstructed human pigmented epidermis (3D-RHPE). Firstly, we reexamined the cytotoxic ranges of CE extract on NHEM, because the B16F10 cells are a murine tumor (melanoma) cell line used for various research in melanogenesis, and therefore it was suspected that the cytotoxic effect of the extract would be different between B16F10 tumor cells and normal human melanocytes. As expected, we found that low cytotoxicity was shown below $5 \mu \mathrm{g} / \mathrm{ml}$ of the extract on NHEM. Using a non-cytotoxic dose, we further confirmed the anti-pigmentation effect of the extract and found that the intracellular melanin levels were significantly decreased in NHEM treated with the extract. In addition, we constructed the 3D-RHPE using normal human keratinocytes and NHEM and tested the effect of the extract on melanogenesis. Fontana-Masson-based melanin staining analysis in the NHEM and 3D-RHPE models showed that melanin production was significantly decreased by treatment with the extract, suggesting that the anti-pigmentation effect of the extract was not based on cell type- and cultured condition-specific results. Therefore, our findings indicate that the $\mathrm{CE}$ extract may be useful for the treatment of hyperpigmentation and for the development of related cosmeceutical products.

In summary, we have provided the first demonstration that extract from the microalga $C$. reinhardtii has an antipigmentation effect in melanocytes. We confirmed that the extracts inhibited $\alpha-\mathrm{MSH}$-induced melanin synthesis and Tyrosinase expression in murine B16F10 cells. Further experiments found that the extract decreased the expression levels of Tyrosinase, TYRP-1 and TYPR-2 mRNAs, and these results were linked to the downregulation of MITF, which is the main transcription factor for those genes. In addition, the extract-induced MITF downregulation was mediated through inhibition of $\alpha$-MSH-induced PKA/CREB and ERK signaling pathways. Using NHEM and 3D-RHPE models, we also confirmed that the extract showed an anti-pigmentation effect. Since carotenoids are known as anti-pigmentation agents, we determined the carotenoid compositions using the HPLC method and found that the extracts had several carotenoids including neoxanthin, violaxanthin, lutein and $\beta$-carotene. Although future additional in-depth studies and clinical evaluations are needed to validate the effect of $C$. reinhardtii extract in human skin, data from the present study have provided novel information on $C$. reinhardtii-mediated anti-pigmentation in melanocytes.

\section{Conflict of Interest}

The authors have no financial conflicts of interest to declare.

\section{Acknowledgments}

This paper was supported by Konkuk University in 2017. 


\section{References}

1. Wasmeier C, Hume AN, Bolasco G, Seabra MC. 2008. Melanosomes at a glance. J. Cell Sci. 121: 3995-3999.

2. Slominski A, Tobin DJ, Shibahara S, Wortsman J. 2004. Melanin pigmentation in mammalian skin and its hormonal regulation. Physiol. Rev. 84: 1155-1228.

3. Kadekaro AL, Chen J, Yang J, Chen S, Jameson J, Swope VB, et al. 2012. Alpha-melanocyte-stimulating hormone suppresses oxidative stress through a p53-mediated signaling pathway in human melanocytes. Mol. Cancer Res. 10: 778-786.

4. Passeron T, Mantoux F, Ortonne JP. 2005. Genetic disorders of pigmentation. Clin. Dermatol. 23: 56-67.

5. Schallreuter KU, Kothari S, Chavan B, Spencer JD. 2008. Regulation of melanogenesis--controversies and new concepts. Exp. Dermatol. 17: 395-404.

6. Naish-Byfield S, Riley PA. 1998. Tyrosinase kinetics: failure of acceleration in oxidation of ring-blocked monohydric phenol substrate. Pigment Cell Res. 11: 94-97.

7. Chan C-F, Huang C-C, Lee M-Y, Lin Y-S. 2014. Fermented broth in tyrosinase- and melanogenesis inhibition. Molecules 19: 13122-13135.

8. Bentley NJ, Eisen T, Goding CR. 1994. Melanocyte-specific expression of the human tyrosinase promoter: activation by the microphthalmia gene product and role of the initiator. Mol. Cell. Biol. 14: 7996-8006.

9. Bonaventure J, Domingues MJ, Larue L. 2013. Cellular and molecular mechanisms controlling the migration of melanocytes and melanoma cells. Pigment Cell Melanoma Res. 26: 316-325.

10. Shibahara S, Yasumoto $K$, Amae S, Udono T, Watanabe $K$, Saito $H$, et al. 2000. Regulation of pigment cell-specific gene expression by MITF. Pigment Cell Res. 13 (Suppl) 8: 98-102.

11. Wang HM, Chen CC, Huynh P, Chang JS. 2015. Exploring the potential of using algae in cosmetics. Bioresour. Technol. 184: 355-362.

12. Zhao X, Xue C-H, Li Z-J, Cai Y-P, Liu H-Y, Qi H-T. 2004. Antioxidant and hepatoprotective activities of low molecular weight sulfated polysaccharide from Laminaria japonica. J. Appl. Phycol. 16: 111-115.

13. Kosourov S, Tsygankov A, Seibert M, Ghirardi ML. 2002. Sustained hydrogen photoproduction by Chlamydomonas reinhardtii: effects of culture parameters. Biotechnol. Bioeng. 78: 731-740.

14. Guedes AC, Amaro HM, Malcata FX. 2011. Microalgae as sources of carotenoids. Marine Drugs 9: 625-644.

15. Lee JJ, An S, Kim KB, Heo J, Cho DH, Oh HM, et al. 2016. Extract of Ettlia sp. YC001 exerts photoprotective effects against UVB irradiation in normal human dermal fibroblasts. J. Microbiol. Biotechnol. 26: 775-783.

16. Heo J, Shin D-S, Cho K, Cho D-H, Lee YJ, Kim H-S. 2018. Indigenous microalga Parachlorella sp. JD-076 as a potential source for lutein production: optimization of lutein productivity via regulation of light intensity and carbon source. Algal Res. 33: 1-7.

17. D'Mello S, Finlay G, Baguley B, Askarian-Amiri M. 2016. Signaling pathways in melanogenesis. Int. J. Mol. Sci. 17: E1144.

18. Shimoda H, Tanaka J, Shan SJ, Maoka T. 2010. Antipigmentary activity of fucoxanthin and its influence on skin mRNA expression of melanogenic molecules. J. Pharm. Pharmacol. 62: 1137-1145.

19. Camera E, Mastrofrancesco A, Fabbri C, Daubrawa F, Picardo M, Sies H, et al. 2009. Astaxanthin, canthaxanthin and beta-carotene differently affect UVA-induced oxidative damage and expression of oxidative stress-responsive enzymes. Exp. Dermatol. 18: 222-231.

20. Ichihashi $M$, Ueda $M$, Budiyanto A, Bito T, Oka M, Fukunaga $\mathrm{M}$, et al. 2003. UV-induced skin damage. Toxicology 189: 21-39.

21. Fiedor J, Burda K. 2014. Potential role of carotenoids as antioxidants in human health and disease. Nutrients 6: 466-488.

22. Dai J, Mumper RJ. 2010. Plant phenolics: extraction, analysis and their antioxidant and anticancer properties. Molecules 15: 7313-7352.

23. Rasouli H, Farzaei MH, Khodarahmi R. 2017. Polyphenols and their benefits: a review. Int. J. Food Prop. 20: 1700-1741.

24. Chang T-S. 2009. An updated review of tyrosinase inhibitors. Int. J. Mol. Sci. 10: 2440-2475.

25. Baek Sh, Lee SH. 2015. Sesamol decreases melanin biosynthesis in melanocyte cells and zebrafish: Possible involvement of MITF via the intracellular cAMP and p38/ JNK signalling pathways. Exp. Dermatol. 24: 761-766.

26. Hartman ML, Czyz M. 2015. MITF in melanoma: mechanisms behind its expression and activity. Cell. Mol. Life Sci. 72: 1249-1260.

27. Burger P, Landreau A, Azoulay S, Michel T, Fernandez X. 2016. Skin whitening cosmetics: feedback and challenges in the development of natural skin lighteners. Cosmetics 3: 36 .

28. Bae-Harboe YS, Park HY. 2012. Tyrosinase: a central regulatory protein for cutaneous pigmentation. J. Invest. Dermatol. 132: 2678-2680.

29. Roh E, Yun C-Y, Yun JY, Park D, Kim ND, Hwang BY, et al. 2013. cAMP-binding site of PKA as a molecular target of bisabolangelone against melanocyte-specific hyperpigmented disorder. J. Invest. Dermatol. 133: 1072-1079.

30. Yun C-Y, Ko SM, Choi YP, Kim BJ, Lee J, Kim JM, et al. 2018. $\alpha$-Viniferin improves facial hyperpigmentation via accelerating feedback termination of cAMP/PKA-signaled phosphorylation circuit in facultative melanogenesis. Theranostics 8: 2031-2043.

31. Jiang Z, Li S, Liu Y, Deng P, Huang J, He G. 2011. Sesamin induces melanogenesis by microphthalmia-associated transcription factor and tyrosinase up-regulation via cAMP signaling pathway. Acta Biochim. Biophys. Sin. 43: 763-770.

32. Hemesath TJ, Price ER, Takemoto C, Badalian T, Fisher DE. 
1998. MAP kinase links the transcription factor Microphthalmia to c-Kit signalling in melanocytes. Nature 391: 298-301.

33. Imokawa G, Yada Y, Kimura M. 1996. Signalling mechanisms of endothelin-induced mitogenesis and melanogenesis in human melanocytes. Biochem. J. 314 (Pt 1): 305-312.

34. Ye Y, Wang H, Chu JH, Chou GX, Yu ZL. 2011. Activation of p38 MAPK pathway contributes to the melanogenic property of apigenin in B16 cells. Exp. Dermatol. 20: 755-757.

35. Saha B, Singh SK, Sarkar C, Bera R, Ratha J, Tobin DJ, et al. 2006. Activation of the Mitf promoter by lipid-stimulated activation of p38-stress signalling to CREB. Pigment Cell Res. 19: 595-605.

36. Yanase $\mathrm{H}$, Ando $\mathrm{H}$, Horikawa $\mathrm{M}$, Watanabe $\mathrm{M}$, Mori $\mathrm{T}$, Matsuda N. 2001. Possible involvement of ERK 1/2 in UVAinduced melanogenesis in cultured normal human epidermal melanocytes. Pigment Cell Res. 14: 103-109.

37. Englaro W, Rezzonico R, Durand-Clément M, Lallemand D, Ortonne JP, Ballotti R. 1995. Mitogen-activated protein kinase pathway and AP-1 are activated during cAMP-induced melanogenesis in B-16 melanoma cells. J. Biol. Chem. 270: 24315-24320.

38. Kim DS, Hwang ES, Lee JE, Kim SY, Kwon SB, Park KC. 2003. Sphingosine-1-phosphate decreases melanin synthesis via sustained ERK activation and subsequent MITF degradation. J. Cell Sci. 116: 1699-1706.

39. Yoon TJ, Lei TC, Yamaguchi Y, Batzer J, Wolber R, Hearing VJ. 2003. Reconstituted 3-dimensional human skin of various ethnic origins as an in vitro model for studies of pigmentation. Anal. Biochem. 318: 260-269.

40. Kandárová $\mathrm{H}$, Liebsch $\mathrm{M}$, Spielmann $\mathrm{H}$, Genschow $\mathrm{E}$, Schmidt E, Traue D, et al. 2006. Assessment of the human epidermis model SkinEthic RHE for in vitro skin corrosion testing of chemicals according to new OECD TG 431. Toxicol. In Vitro 20: 547-559.

41. Kidd DA, Johnson M, Clements J. 2007. Development of an in vitro corrosion/irritation prediction assay using the EpiDerm skin model. Toxicol. In Vitro 21: 1292-1297. 